\title{
Regresando a los valores básicos: las propuestas del Marketing Social y Humanista
}

\section{Regressando aos valores básicos: as propostas do Marketing Social e Humanista}

\author{
JUDITH CAVAZOS-ARROYO* \\ ROGELIO PUENTE-DÍAZ** \\ ANTONIO CARLOS GIULIANI***
}

\section{RESUMEN}

Aunque los planteamientos tradicionales del marketing social han dado resultados razonablemente exitosos, han surgido críticas que están conduciendo a la reflexión de sus paradigmas. Por otro lado, ante las consecuencias desalentadoras de un marketing neoliberal, una perspectiva humano-centrista del marketing empieza a emerger. Este artículo revisó los progresos del marketing social y la reciente propuesta del marketing humanista. Los resultados muestran que ambos postulados convergen y que el marketing social puede integrar la propuesta del marketing humanista. Sin embargo, intervenciones del marketing sustentadas en la práctica de los altos valores que sostiene el marketing humanista, pueden contribuir a la reducción de la desigualdad, mejorar el bienestar y la coexistencia humana, satisfacer con dignidad las necesidades humanas elementarles y hacer más eficiente la articulación de los sistemas de intercambio.

Palabras clave: Marketing social; marketing humanista; valores; bienestar.

* Profesora e investigadora en el Centro Interdisciplinario de Posgrados, de la Universidad Popular Autónoma del Estado de Puebla. Correo electrónico: cavazosjudith03@gmail.com

* Profesor e investigador de la Facultad de Economía y Negocios, de la Universidad Anáhuac México-Norte. Correo electrónico: rogelio.puente@anahuac.mx

*** Profesor e investigador en los Posgrados de Maestría y Doctorado en Administración de la Universidad Metodista de Piracicaba, Brasil. Correo electrónico: cgiuliani@unimep.br 


\section{Abstract}

Although traditional approaches to social marketing have produced reasonable successful results, criticisms have emerged that are leading to the reflection of their paradigms. On the other hand, given the discouraging consequences of neoliberal marketing, a human-centric marketing perspective begins to emerge. This article reviewed the progress of social marketing and the recent approach of humanistic marketing. The results show that both postulates converge and that social marketing can integrate the humanistic marketing perspective. However, marketing interventions based on the practice of the high values of humanistic marketing can contribute to reducing inequality, improving human well-being and coexistence, satisfying basic human needs with dignity, and making a more efficient articulation of exchange systems.

Keywords: Social marketing; humanistic marketing; values; well-being.

\section{INTRODUCCIÓN}

El marketing social fue desarrollado como una aproximación estratégica que facilita el cambio social a través de la adecuación de las herramientas del marketing (KOTLER; ZALTMAN, 1971). Desde su conceptualización y su desarrollo se han establecido principios, conceptos y teorías que han fortalecido la naturaleza y el alcance del marketing social para conducir los procesos de cambio requeridos para lograr un cambio de comportamiento (HASTINGS, 2003; PEATTIE; PEATTIE, 2003; RUSSEL-BENNETT; WOOD; PREVITE 2013). Sin embargo, nuevas perspectivas se debaten para consolidar el marketing social y su efectividad en el cambio de comportamiento de una audiencia determinada (SPOTSWOOD et al., 2012; GORDON; GURRIERI, 2014; LUCA; HIBBERT; MACDONALD, 2016).

Por otro lado, el marketing humanista surge recientemente como una propuesta hacia el desarrollo de bienestar humano por medio de una reconfiguración de las herramientas del marketing tradicional implicando en la satisfacción de necesidades y deseos de los consumidores, la creación de valor sostenible pero también significativo, la transformación y dignidad humana (PIRSON; VAREY, 2014), así como el incremento de la felicidad individual y colectiva 
(NEDELEA; NEDELEA, 2016). Entre las nuevas propuestas, la perspectiva humano-centrista representa una nueva visión que necesita aún ser explorada en la dinámica y complejidad del marketing social y su aportación en la creación de valor social.

Dado que estas ideas y sus implicaciones para el marketing social aún no han sido exploradas a detalle, en este artículo revisamos los progresos del marketing social y la reciente propuesta del marketing humanista, orientados a contribuir a cambios individuales y colectivos más eficientes y significativos.

\section{MARKETING SOCIAL}

El marketing comercial es una función de la organización que implica un grupo de procesos para crear, comunicar y entregar valor en la relación con el cliente, de manera que beneficie a la organización, sus accionistas y públicos. Este enfoque de gestión se sitúa en las escuelas de pensamiento del marketing denominadas como escuelas económicas (SHETH; GARDNER; GARRETT, 1988). Además de estas escuelas, se han desarrollado otras, catalogadas como no económicas vinculándose más estrechamente en las ciencias del comportamiento como la psicología y la sociología (SHETH; GARDNER, GARRETT, 1988). El marketing social se suscribe en lo que Hunt (2011) denominó los sectores micro y macro "no lucrativos", y lo que Sheth, Gardner y Garrett (1988) consideran como el dominio de las escuelas no económicas-interactivas, principalmente dentro de la propuesta del intercambio social.

Existen varias definiciones sobre lo que es el marketing social (GRAHAM, 1997; KOTLER; LEE, 2008). Las perspectivas más recientes lo conceptualizan como un proceso de intercambio entre las unidades sociales, que abre una ventana para aplicar los conocimientos del marketing a través de programas para la creación, comunicación y entrega de valor a fin de influir en los comportamientos de una audiencia, generando con ello el cambio social y beneficiando tanto a la audiencia meta como a la sociedad (KOTLER; LEE, 2008; WYMER, 2011). Una definición consensada por varias asociaciones de marketing social sostiene que busca el desarrollo y la integración de conceptos de marketing con otras aproximaciones para influir en 
comportamientos que beneficien a individuos y comunidades para un mayor bien social (RUNDLE-THIELE, 2015).

Desde la perspectiva de Storey, Saffitz y Rimon (2008), el marketing social es relevante porque implica: 1) desde una perspectiva de mercado, la adopción de una orientación al consumidor, es decir la comprensión y la satisfacción de las necesidades de la audiencia a ser atendida, 2) la comunicación eficiente de la información desde el funcionamiento de los mercados en relación a productos sociales disponibles, costo, utilización, beneficios que proporcionan y dónde obtenerlos y 3) el enfrentamiento de los beneficios de los productos sociales sobre conductas competidoras que operan en un mercado dinámico de ideas, prioridades y diferentes opciones y por tanto, se requiere del desarrollo de estrategias que incrementen el valor percibido de la adopción de una conducta beneficiosa a nivel personal, interpersonal, comunitaria o social.

El paradigma del marketing social se centra principalmente en el bienestar personal y social (KOTLER; ZALTMAN, 1971) y el cambio social (ANDREASEN, 2002). Aun cuando los críticos recientemente están llamando a la reflexión de las bases teóricas (RUNDLE-THIELE, 2015) y la evolución de este campo (LUCA et al., 2016), aún prevalece la propuesta de criterios que se constituyen en el plan de marketing social para lograr el cambio y el bienestar social. Por ejemplo, Andreasen (2002) incluye seis criterios: 1) un objetivo establecido a partir de un cambio de comportamiento, 2) investigación de audiencias para su comprensión durante el diseño, prueba y monitoreo, 3) segmentación de audiencias para asegurar la máxima eficiencia y efectividad, 4) creación de intercambios atractivos y motivacionales con audiencia(s) meta, 5) establecimiento de la mezcla de marketing social (no solamente comunicación o publicidad) y 6) conciencia de la competencia enfrentada para el comportamiento deseado. En general, los expertos en marketing social suelen coincidir con estos criterios porque su aplicación ha sido razonablemente exitosa (LEFEBVRE; FLORA, 1988; STEAD et al., 2007), aunque algunos consideran que aún es necesario comprender cómo el cambio de comportamiento puede ser maximizado para beneficiar a una audiencia o comunidad determinada (RUNDLE-THIELE, 2015) y mejorar la adecuación del marketing social a los 
contextos de cambio social (PEATTIE; PEATTIE, 2003; GORDON; GURRIERI, 2014).

Aunque el enfoque y las prácticas del marketing social son aceptados para una cantidad importante de académicos y practicantes, no está exenta de crítica. Por ejemplo, algunos investigadores (CRAWSHAW, 2012) sugieren que la aproximación actual del marketing social se sustenta en una visión neoliberal que atribuye el bienestar en los individuos, minimizando tanto los factores contextuales, que muchas veces son determinantes del comportamiento individual, como las tensiones de un mundo cada vez más interdependiente y que es donde el marketing social actúa. Por tanto, para lograr el cambio de un comportamiento social debería considerarse (BRENKERT, 2002; PEATTIE; PEATTIE, 2003, GORDON, 2012; LUCA et al., 2016): 1) una agenda de cambio social más amplia, 2) mayor diversidad de teorías de cambio de comportamiento a las utilizadas tradicionalmente, 3) intercambios, interacciones y relaciones sociales complejos (más allá de relaciones diádicas, p.e. formación de redes e interactividad), 4) conflictos entre el bienestar social y las preferencias particulares, 5) fallas del mercado, 6) paradigmas diferentes a la segmentación tradicional (p.e. énfasis en la demografía) y modelos más progresivos a las 4Ps (p.e. circunstancias, capacidad, procesos, relaciones y comunidad) y 7) incorporación de nuevas perspectivas y conceptos.

\section{MARKETING HUMANISTA}

Recientemente emergió el concepto de marketing humanista el cual propone la generación del bienestar humano desde la satisfacción de necesidades y deseos, el rediseño del sistema de prosperidad hacia la creación de valor sustentado en lo auténtico, sostenible y significativo, y, la contribución a la innovación del sistema socio-económico desde la transformación humana al mismo tiempo que se protege la dignidad humana (PIRSON; VAREY, 2014). Nedelea y Nedelea (2016) lo describen como un marketing subordinado a los principios humanistas, que estimula la creación de productos y servicios que son realmente necesarios, proporcionando bienestar y en ningún caso, causan un daño. Por tanto, los autores consideran que el marketing humano representa una nueva visión de la relación entre los individuos y la sociedad en la que viven. 
Las bases del marketing humanista se asientan en la filosofía humanista, la cual toma a los individuos humanos como punto de partida y enfatiza la capacidad humana para razonar. El humanismo asume que la naturaleza humana no está enteramente dada, sino que puede ser refinada a través de la educación, el aprendizaje (PIRSON; VAREY, 2014), la propia capacidad simbólica del hombre para interpretar el mundo, organizar el conjunto de su cultura y construir el sentido de la vida más allá de lo individual, privilegiando lo colectivo (SOLARES, 2011). Los últimos tres siglos se caracterizaron por una importante explosión intelectual de filósofos, científicos y artistas y desde la perspectiva de Gleiser (2014), el siglo XXI trae consigo al ciudadano planetario junto con la oportunidad de desarrollar el humanocentrismo. Esto implica que la ciencia ponga al hombre de vuelta al centro del universo bajo una nueva ética global y la adopción de una nueva relación con el planeta. En este sentido, el argumento central versa en que la vida inteligente en el universo se precisa tan rara y distante, que es necesario celebrar y respetar su existencia y para lograrlo, es necesario desarrollar una nueva moralidad sustentada en la igualdad de todas las criaturas y la preservación de la vida del planeta (GLEISER, 2014).

Algunos investigadores están explorando las posibilidades del desarrollo de propuestas basadas en la intersección entre economía, psicología y humanidades como conductores de la prosperidad en el siglo XXI (POPOVIC; POPOVICH, 2012). El paradigma del marketing humanista se plantea a partir de aspectos involucrados con bienestar, dignidad, integridad (PIRSON; VAREY, 2014) y paz (NEDELEA; NEDELEA, 2016).

\section{Bienestar}

Desde el paradigma del marketing organizacional, el bienestar implica el uso de mecanismos organizacionales basados en el principio de beneficencia y orientados hacia el bienestar de distintos grupos de interés vinculados con la firma (YU; LEE; SIRGY, 2014). La beneficencia es uno de los cinco principios éticos (autonomía, no maleficencia, justicia, fidelidad, beneficencia) de las profesiones orientadas a prestar ayuda (BEAUCHAMP; CHILDRESS, 1983), como la asistencia social, el apoyo comunitario, la salud y el bienestar 
humano, animal y ambiental e incluso el marketing social. Aunque la beneficencia connota actos de bondad, altruismo, humanidad y amor, desde la perspectiva organizacional se define como la cualidad de hacer el bien a través de procesos, actividades o informaciones positivas, con el fin de ayudar o beneficiar a los otros, fomentando intereses legítimos y de bien común, por lo que se trata de un principio y deber moral que confiere y promueve, siempre que sea posible, el bienestar para los grupos de interés de la organización (BATES, 2004).

El marketing social se vincula significativamente con el bienestar ya que su fin es mejorar el bienestar de la sociedad (CARVALHO; MAZZON, 2015; MANIKAM; RUSSEL-BENNETT, 2016) y el fin último del marketing humanista es que las personas alcancen su potencial humano, aspecto que puede converger con el marketing social (CARVALHO; MAZZON, 2015). Este último se enfoca durante los procesos de cambio en las personas, sus deseos y necesidades, aspiraciones, estilo de vida y libertad de elección (LEFEBVRE, 2011), de hecho, el concepto de bienestar se ha asociado con felicidad y satisfacción con la vida, pero va más allá (CARVALHO; MAZZON, 2015). El denominado marketing del bienestar cobra sentido donde prevalecen las relaciones de largo plazo con audiencias o clientes y se sustenta en la valoración y el aprecio por una mejor calidad de vida (YU; LEE; SIRGY, 2014), seguridad para los diferentes públicos y el medio ambiente, búsqueda del bienestar objetivo y subjetivo de las audiencias y/o consumidores, sin generar condiciones adversas entre los diferentes actores vinculados a la organización y la orientación a metas tanto financieras como sociales (SIRGY; LEE, 2008).

\section{Dignidad}

La noción de dignidad humana ha sido central para el progreso de las sociedades, sin embargo, el concepto resulta complejo de definir (CHOCHINOV, 2012). En general suele explicarse desde dos vertientes que parten de los derechos que tiene todo ser humano, la dignidad intrínseca o moral y la dignidad extrínseca o jurídica. La dignidad intrínseca implica la igualdad del valor de la vida de todos los seres humanos. López (2005) afirma que en su sentido intrínseco la dignidad incluye libertad, autonomía, integridad y respeto. En 
cambio, la dignidad extrínseca involucra la igualdad de derechos y condiciones de vida de todos los seres humanos (PALACIOS; ROMAÑACH, 2007).

La denominada economía de la dignidad (POPOVIC; POPOVI$\mathrm{CH}, 2012$ ) considera la importancia del establecimiento de un vínculo vital entre las relaciones interpersonales y el desarrollo humano en la generación de riqueza. En este sentido, se requeriría de una reconfiguración de los participantes económicos en los procesos de intercambio, donde no solamente se es consumidor, productor o intermediario, sino un participante activo de una comunidad cuyo valor agregado no se sustenta solamente en el precio y en el valor de uso, sino también en empoderamiento, participación, contribución, proactividad, auto-realización, sentido de co-responsabilidad y reciprocidad en la creación de riqueza en condiciones de dignidad y libertad que propicien el desarrollo humano, estimulen su potencial y su plenitud. Popović y Popovich (2012) consideran esto como dominio de la estima humana desde un espectro individual y colectivo de naturaleza económica, social, psicológica y espiritual a fin de honrar el potencial humano al máximo.

Aunque la dignidad debe ser un aspecto clave de todo tipo de marketing, una posibilidad viable para su aplicación en marketing es desde el paradigma de un marketing social transformador de los sistemas sociales. Lefebvre (2012) propone un modelo tridimensional del marketing social transformador que considera: el alcance del cambio desde el intercambio, el diseño de los programas y servicios sociales orientados a resolver los problemas sociales y el espacio de valores que deben integrarse a cualquier programa de marketing social. El autor considera que el alcance facilita los intercambios a través de co-creación, conversación, comunidad y mercados; que todo diseño de programas y servicios en marketing social debe honrar a la gente, radiar la propuesta de valor, comprometerse con el servicio y mejora de las experiencias e integrar todo programa de marketing social con cuatro valores: dignidad, esperanza, amor y confianza. Existen varios modelos recientes de marketing, emprendimiento e innovación sociales con aplicaciones creativas de sistemas que están abriendo oportunidades de impacto social significativo bajo dinámicas de interacciones interdisciplinarias transformadoras (DAHAN et al., 2010). 


\section{Integridad}

Se describe como un estado sofisticado de procesamiento de la experiencia que abarca moral, juicio, creatividad, capacidad intuitiva y poder analítico-racional (FERGUSON, 2009). En la comercialización generalmente la integridad transmite dos significados. Uno de ellos se refiere a la práctica ética de la gestión del marketing al tomar decisiones y realizar comportamientos, enfrentándose con ello a tensiones normativas y posibles violaciones éticas y, el otro enfoque más académico involucra integrar la teoría del marketing en la óptica de la ética del marketing (ABELA; MURPHY, 2007).

La organización humanista visualiza la integridad como un factor productivo, una capacidad dinámica que alinea, forma, extiende o modifica recursos y procesos organizacionales para generar mayores niveles de desempeño (DRNEVICH; KRIAUCIUNAS, 2011; FUERST; SCHOTTER, 2011). Por tanto, este tipo de organización requiere ser proactiva en la integración de procesos y estructuras dinámicas de la gestión de la integridad (JENNINGS, 2006), capaz de crear un vínculo entre la propia integridad, el incremento del desempeño, la calidad de vida y la creación de valor para todos los actores involucrados con ella (ERHARD; JENSEN, 2014). Además, la integridad organizacional requiere apertura a las influencias externas para negociar y comprometerse con los otros, compromiso con uno mismo, sentido de membresía, lealtad compatible con los valores personales y sentido de autonomía moral (FERGUSON, 2009).

Erhard, Jensen y Zaffron (2009) proponen un modelo de integridad que abarca honrar la propia integridad, así como las normas morales, éticas y legales teniendo como dominio de aplicación personas, grupos, organizaciones, sociedad, naciones e incluso objetos y sistemas, incidiendo en el trabajo y la honradez y consecuentemente, en el desempeño. La integridad social y cultural puede ser vinculada con la sustentabilidad y la capacidad de generar capital social para hacer frente a los problemas sociales, económicos y ambientales de un sistema, por lo tanto, se relaciona con el bienestar humano (HEDIGER, 2000). Se espera que los programas de marketing social tengan un impacto social positivo, basándose en aspectos como honestidad, confianza y buena reputación, desde su propia dinámica 
y estimulados a través de las interacciones con los distintos agentes del micro y mesoentorno.

\section{$\operatorname{Paz}$}

Organizaciones no gubernamentales y gubernamentales suelen colaborar en la promoción de la paz y la reconciliación para reducir conflictos (KOLK; LENFANT, 2015). Sin embargo, recientemente también se ha dado la inclusión de la construcción y la promoción de estrategias de paz duradera desde las organizaciones lucrativas (NELSON, 2000). Estas suelen aprovecharla como un mecanismo de participación de responsabilidad social empresarial que además de promover un cambio social positivo, responde a los intereses económicos de la organización (RETTBERG, 2016). Por ejemplo, Lazer (1969) afirma que uno de los papeles sociales del marketing corresponde a la búsqueda de la paz, ya que sin ella no es posible el progreso económico.

Cualquier organización que asuma la paz como un estandarte estratégico requiere colocar en el centro a los seres humanos involucrados en el proceso de paz y gestionar a partir de ello recursos, actividades, negociaciones o alianzas, a fin de lograr interacciones progresivas con mercados e instituciones que generen resultados benéficos, profundos y perdurables (KOLK; LENFANT, 2016). Nedelea y Nedelea (2016) argumentan que el marketing también puede trasladarse del paradigma organizacional al ámbito del humanismo. Los investigadores vinculan al marketing humanista con el marketing de la paz a través de la necesidad de la promoción de los valores y las aspiraciones fundamentales a la interioridad del ser humano.

Dado que existe una relación interactiva entre el desarrollo social, la paz y los derechos humanos (WEERAWARDENA; SULLIVAN-MORT, 2006), el marketing de la paz reafirma la fe en los derechos humanos fundamentales, en la dignidad, el valor de la persona humana y la igualdad a los derechos entre los seres humanos, considera que la práctica de la tolerancia, paz y seguridad como intereses en común de todas las personas promueven el progreso social y económico que mejoran las condiciones de vida (NEDELEA; NEDELEA, 2016). Sin embargo, la construcción de la paz es incremental y de largo plazo y requiere que las or- 
ganizaciones transformen gradualmente sus prácticas culturales (KATSOS; FORT, 2016).

\section{DISTINCIÓN ENTRE EL MARKETING SOCIAL Y HUMANISTA}

A partir de los planteamientos del marketing social y humanista se realizó un análisis comparativo de sus características fundamentales (Tabla 1). Los resultados muestran la madurez del marketing social como paradigma (SPOTSWOOD et al., 2012) y la incipiente configuración del marketing humanista (PIRSON; VAREY, 2014), el cual refleja desde las particularidades del humanismo, parte de los propósitos del marketing social. El marketing social se encuentra en una etapa de debate, enriquecimiento de perspectivas y adaptaciones de herramientas del marketing comercial a la resolución eficiente de problemas sociales (LEFEBVRE, 2012; SPOTSWOOD et al., 2012).

El enfoque tradicional del marketing social se ha centrado en el cambio comportamental y el desarrollo de estrategias a partir de una adaptación de las denominadas 4Ps, pero los replanteamientos recientes están incluyendo reflexiones y propuestas a partir del marketing crítico (SAREN et al., 2007), el marketing transformacional (MICK et al., 2012), la lógica dominante de servicios y la co-creación del valor social (LEFEBVRE, 2012). La naturaleza del marketing social es profundamente humana, por tanto, da cabida en su marco al enfoque, medios y alcances del marketing humanista. Sin embargo, el desarrollo de procesos y estrategias desde el humanismo, así como su aplicación en diferentes contextos enriquecerá a ambos enfoques, trayendo nueva luz sobre mecanismos y herramientas para lograr el bienestar humano. 
Tabla 1: Comparación entre marketing social y humanista

\begin{tabular}{|c|c|c|}
\hline Característica & Marketing social & Marketing humanista \\
\hline Propósitos & $\begin{array}{l}\text { Bienestar indi- } \\
\text { vidual y social } \\
\text { Cambio social positivo }\end{array}$ & $\begin{array}{l}\text { Bienestar humano } \\
\text { Incremento de la felicidad } \\
\text { individual y colectiva } \\
\text { Logro del potencial humano }\end{array}$ \\
\hline Principios & $\begin{array}{l}\text { Sociales, culturales, } \\
\text { psicológicos y democrá- } \\
\text { ticos }\end{array}$ & Humanistas \\
\hline Enfoque & $\begin{array}{l}\text { Necesidades de los } \\
\text { individuos, sociales o } \\
\text { de ambos } \\
\text { Solución sostenible de } \\
\text { problemas sociales } \\
\text { Mercado para cambiar } \\
\text { comportamientos e } \\
\text { ideas }\end{array}$ & $\begin{array}{l}\text { Necesidades y deseos huma- } \\
\text { nos fundamentales } \\
\text { Desarrollo del potencial y la } \\
\text { plenitud humanas } \\
\text { Mercado para desarrollar y } \\
\text { cambiar valores }\end{array}$ \\
\hline $\begin{array}{l}\text { Medios para } \\
\text { lograr el obje- } \\
\text { tivo }\end{array}$ & $\begin{array}{l}\text { Procesos de cambio } \\
\text { comportamental de una } \\
\text { audiencia } \\
\text { Plan de marketing } \\
\text { social } \\
\text { Transformación indivi- } \\
\text { dual y social } \\
\end{array}$ & $\begin{array}{l}\text { Desarrollo y enaltecimiento } \\
\text { de valores humanos en los } \\
\text { actores involucrados } \\
\text { Transformación humana }\end{array}$ \\
\hline $\begin{array}{l}\text { Proposición de } \\
\text { valor }\end{array}$ & $\begin{array}{l}\text { Creación y entrega } \\
\text { de valor individual y } \\
\text { social transformador } \\
\text { generando beneficios } \\
\text { personales y sociales } \\
\text { sostenibles } \\
\end{array}$ & $\begin{array}{l}\text { Creación de valores sosteni- } \\
\text { bles de forma significativa y } \\
\text { transformadora generando } \\
\text { bienestar personal y colecti- } \\
\text { vo sostenibles }\end{array}$ \\
\hline $\begin{array}{l}\text { Intercambio } \\
\text { para la crea- } \\
\text { ción de valor }\end{array}$ & $\begin{array}{l}\text { Cambio de una idea o } \\
\text { comportamiento por } \\
\text { otro en la audiencia } \\
\text { Co-creación, conver- } \\
\text { saciones, relaciones y } \\
\text { redes }\end{array}$ & $\begin{array}{l}\text { Desarrollo o cambio de valo- } \\
\text { res humanos en el target }\end{array}$ \\
\hline
\end{tabular}




\begin{tabular}{|c|c|c|}
\hline $\begin{array}{l}\text { Escuela de } \\
\text { pensamiento }\end{array}$ & No económica-interactiva & No económica-interactiva \\
\hline $\begin{array}{l}\text { Aplicaciones } \\
\text { económicas }\end{array}$ & $\begin{array}{l}\text { Escuela económica-interactiva } \\
\text { Marketing Relacionado con Cau- } \\
\text { sas (MRC) } \\
\text { Responsabilidad social empre- } \\
\text { sarial } \\
\text { Marketing interno } \\
\text { Economía circular } \\
\text { Valor compartido }\end{array}$ & $\begin{array}{l}\text { Escuela económica-interactiva } \\
\text { Marketing Relacionado con Cau- } \\
\text { sas (MRC) } \\
\text { Responsabilidad social empresa- } \\
\text { rial } \\
\text { Marketing interno } \\
\text { Valor compartido }\end{array}$ \\
\hline Target & $\begin{array}{l}\text { Selección de una audiencia pri- } \\
\text { maria }\end{array}$ & $\begin{array}{l}\text { Clientes } \\
\text { Empleados } \\
\text { Públicos interesados (stakeholders) } \\
\text { Audiencia seleccionada }\end{array}$ \\
\hline $\begin{array}{l}\text { Orientación al } \\
\text { mercado }\end{array}$ & Social & Humanista \\
\hline $\begin{array}{l}\text { Proyectos y } \\
\text { programas }\end{array}$ & $\begin{array}{l}\text { Individuales } \\
\text { Alianzas } \\
\text { Stakeholders }\end{array}$ & $\begin{array}{l}\text { Individuales } \\
\text { Alianzas } \\
\text { Stakeholders }\end{array}$ \\
\hline $\begin{array}{l}\text { Desarrollo } \\
\text { estrategias }\end{array}$ & $\begin{array}{l}\text { Mezcla de marketing social } \\
\text { Diseño de servicios bajo la } \\
\text { premisa de generar experiencias } \\
\text { significativas para el aprendizaje } \\
\text { y adquisición de nuevos com- } \\
\text { portamientos }\end{array}$ & $\begin{array}{l}\text { Desde productos y servicios } \\
\text { orientados al bienestar y no dañi- } \\
\text { nos }\end{array}$ \\
\hline Ejes base & $\begin{array}{l}\text { Bienestar social } \\
\text { Desarrollo comunitario y social } \\
\text { Participación ciudadana } \\
\text { Valores } \\
\text { Justicia social } \\
\text { Ética y transparencia } \\
\text { Capital social }\end{array}$ & $\begin{array}{l}\text { Valores humanos } \\
\text { Bienestar humano } \\
\text { Derechos humanos } \\
\text { Desarrollo humano } \\
\text { Aspiraciones humanas } \\
\text { Justicia social } \\
\text { Moral y ética }\end{array}$ \\
\hline $\begin{array}{l}\text { Valores desta- } \\
\text { cados }\end{array}$ & $\begin{array}{l}\text { Dignidad, esperanza, amor y } \\
\text { confianza } \\
\text { Valores democráticos }\end{array}$ & $\begin{array}{l}\text { Privilegia todos los valores huma- } \\
\text { nos } \\
\text { Dignidad, integridad, paz }\end{array}$ \\
\hline Resultados & $\begin{array}{l}\text { A largo plazo } \\
\text { Razonablemente exitosos }\end{array}$ & $\begin{array}{l}\text { A largo plazo } \\
\text { Sin suficientes evidencias }\end{array}$ \\
\hline Desafíos & $\begin{array}{l}\text { Fortalecimiento teórico } \\
\text { Maximización de beneficios } \\
\text { sociales } \\
\text { Adecuación del paradigma a la } \\
\text { complejidad social } \\
\text { Propuestas estratégicas desde } \\
\text { herramientas diferentes a las 4Ps }\end{array}$ & $\begin{array}{l}\text { Desarrollo de las bases teóricas } \\
\text { Desarrollo de un proceso estraté- } \\
\text { gico } \\
\text { Resultados de intervenciones } \\
\text { prácticas exitosas }\end{array}$ \\
\hline
\end{tabular}

Fonte: Elaborada pelos autores (2016). 


\section{Posibilidades DE DESARRollo DEL MARKETING HUMANiSTA}

Las aportaciones de la gestión humanista continúan avanzando (PIRSON; KOSTERA, 2017) y sus postulados comienzan a permear tanto en organizaciones lucrativas, híbridas y no lucrativas (KOLK; LENFANT, 2016). Los pilares básicos del paradigma parten de bases robustas, pero sus aplicaciones en el marketing aún requieren profundizarse teórica y empíricamente en la consideración del elemento humano con la identificación y las interacciones de la organización con diferentes actores en los sistemas de mercado, la re-significación de las relaciones, confianza y compromiso con clientes y stakeholders, credibilidad y transparencia en los procesos y alcances del desarrollo humano.

Por ejemplo, desde el sistema económico, una intervención humanista requiere de un diagnóstico que comprenda profundamente el contexto y a los agentes del mercado implicados en la dinámica del sistema de intercambio y de relaciones con los clientes. En el día a día, los clientes se enfrentan a una gran diversidad de aspectos que limitan su íntegra potencialidad humana, para vivir el presente e idealizar el futuro bajo el paraguas de las necesidades y los deseos individuales y colectivos, así como las imposiciones naturales o sociales que inhiben la capacidad de la plenitud voluntaria con dignidad, igualdad, justicia y libertad (SAREN et al., 2007). Resultados de una investigación en negocios híbridos que integran en su propósito paz, reconciliación y reducción del conflicto en Congo y Ruanda (KOLK; LENFANT, 2016) mostraron que este tipo de organizaciones interactúan progresivamente con mercados e instituciones manteniendo como aspectos clave la paz, la justicia, el desarrollo humano, la reconciliación y la esperanza para las comunidades involucradas con los negocios híbridos.

Aún es necesario el desarrollo de alternativas viables que respondan a una conducción de un marketing que se comprometa siempre con una transformación para el bienestar y el desarrollo humano (NEDELEA; NEDELEA, 2016). En el marketing comercial se han realizado esfuerzos fructíferos que pueden ser ubicados en una intersección con el marketing humanista. Las posibilidades desde la responsabilidad socio-humana empresarial son variadas e implican, 
por ejemplo, intercambios mutuamente benéficos, integridad en las prácticas de gestión comercial, sustentabilidad y responsabilidad social y humana colectiva, desarrollo de la productividad y prosperidad de las comunidades, preservación de interacciones, transacciones y relaciones basadas en dignidad, respeto, paz, esperanza y responsabilidad compartida, así como un consumo consciente orientado a la obtención de valor, a partir de la construcción de significados, experiencias emocionales (persona-persona) más profundas y significativas, dinámica de las relaciones y participación comunitaria (PEATTIE; PEATTIE; THOMAS, 2012).

Algunos autores (BORGERSON, 2014) sostienen que la propuesta actual del marketing humanista aún no cumple con argumentos suficientes y rigurosos para su desarrollo, ya que sus postulados se centran principalmente en la esperanza de un mundo más digno con base a una transformación del mercado capitalista. Sin embargo, tanto el marketing social (SPOTSWOOD et al., 2012) como el comercial (MACLARAN; BROWN, 2005) también han presentado desafíos para su consolidación teórica. Adicionalmente, las interacciones y dinámicas entre los actores del mercado no suelen estar libres de tensiones, por lo que el marketing humanista puede funcionar como una aspiración para un funcionamiento de los sistemas, con menos asimetrías en las prácticas de los sistemas de producción y consumo, a través de mecanismos ético-culturales e incluso como formas de respuesta a modo de resistencia a ciertos sistemas económicos.

La complejidad de los sistemas actuales (LEFEBVRE, 2012) acentúan la necesidad de (re)valorar la naturaleza humana, sus capacidades, derechos y obligaciones. Seguramente, la experiencia de "ser humano" se verá aún más vinculada a interacciones que requerirán una mayor comprensión tanto biológica como filosófica de lo que significa ser humano (SHARON, 2014) y, el pensamiento mercadológico no escapa de esta transcendental tarea. Para las personas, en sus roles de colaboradores, clientes y ciudadanos, el paradigma humanista emerge como una propuesta y una esperanza para abordar necesidades, deseos y relaciones con los agentes sociales con los que interactúan desde un enfoque distinto. 


\section{REFLEXIONES FINALES}

Probablemente varios académicos y practicantes del marketing integrarán la propuesta del marketing humanista dentro del paradigma del marketing social, lo cual es posible porque sus postulados convergen. No obstante, la adopción de una perspectiva humanista, por su propia naturaleza trastoca la esencia de una organización y particularmente el marketing humanista, requiere una reconceptualización de paradigmas, desarrollo de nuevas estrategias y mecanismos con los que se operan los modelos de obtención y (re) distribución de beneficios.

El desarrollo de una visión humanista se sustenta en una visión integral que aspira a la práctica de altos valores, por tanto, requiere de una profunda reeducación de todos los actores del mercado involucrados, a fin de hacer más equitativas las relaciones de intercambio que suelen servir asimétricamente sobre los intereses de una minoría. La solución no consiste sencillamente en eliminar los abusos, sino en transformar tanto la forma en que se organiza la estructura social (HORKHEIMER, 1972), como la ejecución de las interacciones entre las redes del sistema de mercado.

Por ejemplo, Latinoamérica continúa siendo una de las regiones con mayores desigualdades en el mundo. Aunque estos países mantienen la búsqueda y la aplicación de políticas y mecanismos que contribuyan a mantener su crecimiento económico y que disminuyan la marcada desigualdad entre sus ciudadanos, hasta ahora los resultados han sido limitados (NAVARRO, 2014). Es probable que si se regresa a modelos autoritarios no se alcanzará la libertad, dignidad y justicia que tan urgentemente necesita la región, sino que se requieren propuestas estratégicas sustentadas en las ciencias sociales y las humanidades para superar la inmensa y dolorosa deuda social latinoamericana, fincada en la corrupción, la crueldad, la exclusión y la desesperanza (SOSA, 2011).

Aun si el marketing humanista es un ideal, empresarios, profesionales y académicos asumirán sus argumentos y propuestas en la medida en que los consumidores valoren lo que puede ofrecer, desarrollándose paralelamente a las implementaciones científicas y tecnológicas que se asumen como parte de la cotidianidad. En última instancia, los seres humanos independientemente de nues- 
tros diferentes roles y aspiraciones, tenemos la corresponsabilidad de mejorar nuestra coexistencia humana en la sociedad en la que convivimos, apropiarnos de la tarea de la articulación de los sistemas de intercambio, de la satisfacción digna e íntegra de las necesidades más elementales, del desarrollo humano y el bienestar de nuestra humanidad.

\section{REFERENCIAS}

ABELA, A. V.; MURPHY, P. E. Marketing with integrity: ethics and the service-dominant logic for marketing. Journal of the Academy of Marketing Science, v. 36, n. 1, p. 39-53, 2007.

ANDREASEN, A. R. Marketing social marketing in the social change marketplace. Journal of Public Policy \& Marketing, Chicago, v. 21, n. 1, p. 3-13, 2002.

BATES, R. Critical analysis of evaluation practice: the Kirkpatrick model and the principle of beneficence. Evaluation and Program Planning, v. 27, n. 3, p. 341-7, 2004.

BEAUCHAMP, T. L.; CHILDRESS, J. F. Principles of biomedical ethics. New York: Oxford University Press, 1983.

BORGERSON, J. L. Humanistic marketing. Consumption, Markets \& Culture, v. 18, n. 2, p. 1-7. jul./2014.

BRENKERT, G. G. Ethical challenges of social marketing. Journal of Public Policy \& Marketing, v. 21, n. 1, p. 14-25, 2002.

CARVALHO, H. C.; MAZZON, J. A. A better life is possible: the ultimate purpose of social marketing. Journal of Social Marketing, v. 5, n. 2, p. 169-186, 2015.

CRAWSHAW, P. Governing at a distance: Social marketing and the (bio) politics of Responsibility. Social Science \& Medicine, v. 75, p. 200-7, 2012.

CHOCHINOV, H. M. Dignity Therapy. New York: Oxford University Press, 2012.

D A HAN, N. et al. Corporate-NGO Collaboration: co-creating new business models for developing markets. Long Range Planning, v. 43, n. 2-3, p. 326-42, 2010.

DRNEVICH, P. L.; KRIAUCIUNAS, A. P. Clarifying the conditions and limits of the contributions of ordinary and dynamic capabilities to relative firm performance. Strategic Management Journal, v. 32, n. 3, p. 254-279, 2011.

ERHARD, W.; JENSEN, M. Beyond Agency Theory: The Power of Integrity. Harvard Business School, NOM Research Paper n. 10-06; Barbados Group Working Paper no. 10-02; Negotiation, Organizations and Markets. p. 1-58, 2014. Desde: <http://papers.ssrn.com/sol3/ Papers.cfm?abstract_id=2432084>. Acceso en: 15 jul. 2016.

ERHARD, W.; JENSEN M. C.; ZAFFRON, S. Integrity: A Positive Model that Incorporates the Normative Phenomena of Morality, Ethics and Legality. Harvard Business School, NOM 2009. 
FERGUSON, B. Creativity and Integrity: Marketing the "In Development" Screenplay. Psychology \& Marketing. v.26, n.5, p.421-444, 2009.

FUERST, M.; SCHOTTER, A. Strategic integrity management as a dynamic capability. In: WILKINSON, T. (Ed.). Strategic Management in the 21st Century. Praeger Publishers. 2011. Desde: <http://ssrn.com/abstract=2064981>. Acceso en: 13 jul. 2016.

GLEISER, M. O iluminismo e o humanocentrismo. Folha de S.Paulo, 6 jul. 2014. Desde: < http://www1.folha.uol.com.br/colunas/marcelogleiser/2014/07/1481766-o-iluminismo-e-o-humanocentrismo.shtml >. Acceso en: 15 jul. 2016.

GORDON, R. Re-thinking and Re-tooling the Social Marketing Mix. Australasian Marketing Journal, v. 20, n. 2, p. 122-6, 2012.

GORDON, R.; GURRIERI, L. Towards a reflexive turn: Social marketing assemblages. Journal of Social Marketing, n. 4, v. 3, p. 261-278, 2014.

GRAHAM, J. L. Marketing social change: Changing behavior to promote health, social development, and the environment. Journal of Marketing Research, v. 34, n. 2, p. 294-301, 1997.

HASTINGS, G. Relational paradigms in social marketing. Journal of Macromarketing, v. 23, n. 1, p. 6-15, 2003.

HEDIGER, W. Sustainable development and social welfare. Ecological Economics, v. 32, n. 3, p. 481-92, 2000.

HORKHEIMER, M. Critical theory: Selected essays. Vol. 1. New York: Bloomsbury Publishing, 1972.

HUNT, S. D. On the intersection of marketing history and marketing theory. Marketing Theory, v. 11, n. 4, p. 483-9, 2011.

JENNINGS, M. The seven signs of ethical collapse: How to spot moral meltdowns in companies... Before it's too late. USA: Macmillan. 2006.

KATSOS, J. E.; FORT, T. L. Leadership in the promotion of peace: Interviews with the 2015 Business for Peace honorees. Business Horizons, v. 59, n. 5, p. 463-70, 2016.

KOLK, A.; LENFANT, F. Partnerships for peace and development in fragile states: Identifying missing links. Academy of Management Perspectives, v. 29, n. 4, p. 422-37, 2015.

. Hybrid business models for peace and reconciliation. Business Horizons, v. 59, n. 5, p. 503-24, 2016.

KOTLER, P.; LEE, N. Social marketing. London: SAGE, 2008.

KOTLER, P.; ZALTMAN, G. Social marketing: An approach to planned social change. Journal of Marketing, v. 35, n. 3, p. 3-12, 1971.

LAZER, W. Marketing's changing social relationships. The Journal of Marketing, v.33, n.1, p. 3-9, 1969.

LEFEBVRE, R. C.; FLORA, J. A. Social marketing and public health intervention. Health Education Quarterly, v. 15, n. 3, p. 299-315, 1988. 
LEFEBVRE, R. C. An integrative model for social marketing. Journal of Social Marketing, v. 1, n. 1, p. 54-72, 2011.

- Transformative social marketing: Co-creating the social marketing discipline and brand. Journal of Social Marketing, v. 2, n. 2, p. 118-24, 2012.

LÓPEZ, M. T. Dignidad, igualdad. La buena política europea. En: Ciudadanos de Europa. Derechos fundamentales en la Unión Europea. Madrid: Biblioteca Nueva, 2005, p. 83-90.

LUCA, N. R.; HIBBERT, S.; MCDONALD, R. Towards a service-dominant approach to social marketing. Marketing Theory, v. 16, n. 2, p. 194-218, 2016.

MACLARAN, P.; BROWN, S. The center cannot hold: Consuming the utopian marketplace. Journal of Consumer Research, v. 32, n. 2, p. 311-23, 2005.

MANIKAM, S.; RUSSELL-BENNETT, R. The social marketing theory-based (SMT) approach for designing interventions. Journal of Social Marketing, v. 6, n. 1, p. 18-40, 2016.

MICK, G. D. et al. Transformative Consumer Research. For Personal and Collective Well-Being. USA: Routledge, 2012.

NAVARRO, V. Las limitaciones de los programas antipobreza. Pensamiento Crítico. 2014. Desde: <http://www.vnavarro.org/wp-content/uploads/2014/02/11-02-14-las-limitaciones-de-los-programas-antipobreza-p024-vdef-100214-edit.pdf>. Acceso en: 15 jul. 2016.

NEDELEA, A. M.; NEDELEA, M. O. Humane Marketing, peace marketing and rebranding marketing. Ecoforum Journal, v. 5, n. 2, p. 288-91, 2016.

NELSON, J. The business of peace. London: Prince of Wales Business Forum, 2000.

PALACIOS, A.; ROMAÑACH, J. El modelo de la diversidad: una nueva visión de la bioética desde la perspectiva de las personas con diversidad funcional (discapacidad). Intersticios, v. 2, n.2, p. 37-47, 2007. Desde: <http://www.diversocracia.org/docs/El_modelo_de_la_diversidad_una_vision_general.pdf $>$. Acceso en: 15 jul. 2016.

PEATTIE, S.; PEATTIE, K. Ready to fly solo? Reducing social marketing's dependence on commercial marketing theory. Marketing Theory, v. 3, n. 3, p. 365-85, 2003.

PEATTIE, S.; PEATTIE, K.; THOMAS, R. Social Marketing as Transformational Marketing in public services. Public Management Review, v. 14, n. 7, p. 987-1010, 2012.

PIRSON, M.; KOSTERA, M. Introduction to Dignity and Organization. En: PIRSON, M.; KOSTERA, M. (Ed.). Dignity and the Organization. London: Palgrave Macmillan, 2017, p. 1-9.

PIRSON, M.; VAREY, R. Introduction. En: VAREY, R. J.; PIRSON, M. (Ed.). Humanistic Marketing. London: Palgrave Macmillan, 2014, p. 1-15.

POPOVIĆ, S.; POPOVICH, L. M. Economics of Dignity: Growing people from consumers to members. Eruditio, v. 1, n. 4, p. 151-6, 2012.

RETTBERG, A. Need, creed, and greed: Understanding why business leaders focus on issues of peace. Business Horizons, v. 59, n. 5, p. 481-92, 2016.

RUNDLE-THIELE, S. Looking back and moving forwards: An agenda for social marketing research. Recherche et Applications en Marketing, v. 30, n. 3, p. 128-33, 2015. 
RUSSELL-BENNETT, R.; WOOD, M.; PREVITE, J. Fresh ideas: services thinking for social marketing. Journal of Social Marketing, v. 3, n. 3, p. 223-38, 2013.

SAREN, M. et al. Introduction: Defining the field of Critical Marketing. En: SAREN, M.; MACLARAN, P.; GOULDING, C.; ELLIOTT, R.; SHANKAR, A.; CATTERALL, M. (Ed.). Critical marketing: Defining the field. Amsterdam: Elsevier, 2007, p. xviii-xxiii.

SHARON, T. Human Nature in an Age of Biotechnology: The Case for Mediated Posthumanism, Philosophy of Engineering and Technology. USA: Springer Science+Business, 2014.

SHETH, J. N.; GARDNER, D. M.; GARRETT, D. E. Marketing theory: evolution and evaluation. V. 1. New York: Wiley, 1988.

SIRGY, J. M.; LEE, D. J. Well-being marketing: An ethical business philosophy for consumer goods firms. Journal of Business Ethics, v. 77, n. 4, p. 377-403, 2008.

SOSA, R. Desigualdad, exclusión y pobreza en América Latina: La inmensa deuda social del neoliberalismo. Revista do Instituto Brasileiro de Direitos Humanos, v. 11, n. 11, p. 155-66, 2011.

SPOTSWOOD, F. et al. Some reasonable but uncomfortable questions about social marketing. Journal of Social Marketing, v. 2, n. 3, p. 163-75, 2012.

STEAD, M. et al. A systematic review of social marketing effectiveness. Health education, v. 107, n. 2, p. 126-91, 2007.

STOREY, J.; SAFFITZM, G.; RIMON, J. Social Marketing. En: GLANZ, K.; RIMER, B.; VISWANATH, K. (Ed.). Health Behavior and Health Education Theory, Research, and Practice. 4th ed. San Francisco: Jossey-Bass, 2008, p. 169-88.

VAREY, R. J.; PIRSON, M. Humanistic Marketing. London: Palgrave, Macmillan, 2014.

WEERAWARDENA, J.; SULLIVAN-MORT, G. Investigating social entrepreneurship: A multidimensional model. Journal of World Business, v. 41, n. 1, p. 21-35, 2006.

WYMER, W. Developing more effective social marketing strategies. Journal of Social Marketing, v. 1, n. 1, p. 17-31, 2011.

YU, G.; LEE, D. J.; SIRGY, J. Well-being Marketing as Humanistic Marketing. En: VAREY, R. J.; PIRSON, M. (Ed.). Humanistic Marketing. London: Palgrave, Macmillan, 2014, p.164-75.

Recebido em: 03-03-2017

Aprovado em: 24-04-2017

Avaliado pelo sistema double blind review.

Editor: Elmo Tambosi Filho

Disponível em http://mjs.metodista.br/index.php/roc 